\title{
Comprehensive Breast Care for the Future from the American Society of Breast Surgeons
}

\author{
Dennis Holmes, $\mathrm{MD}^{1}$, and Henry M. Kuerer, $\mathrm{MD}, \mathrm{PhD}^{2}$ \\ ${ }^{1}$ Department of Surgery, John Wayne Cancer Institute, Santa Monica, CA; ${ }^{2}$ Division of Surgery, Department of Breast \\ Surgical Oncology, The University of Texas MD Anderson Cancer Center, Houston, TX
}

This year, the 20th Annual Meeting of the American Society of Breast Surgeons, held in Dallas, Texas, had an attendance of 1556 people from across 37 countries. A theme seen in many talks at this year's meeting is further preparation for our members to be comprehensive breast care physicians of the future, which includes an emphasis on expertise, treatment innovation, and further minimization of treatment side effects. This year, we committed to helping attendees elevate standard-practice by improving the efficacy, safety, and quality of breast care for our patients.

\section{GENERAL SESSION HIGHLIGHTS}

We were delighted to welcome our keynote speaker, Academy Award-winning actor and lymphedema advocate, Kathy Bates, who eloquently highlighted her compelling personal story of cancer and the society's increased focus on breast cancer-related lymphedema (otherwise increasingly known as LE), including this year's inaugural premeeting course and cadaver lab on Surgical Prevention and Management of Lymphedema. Ms. Bates is an avid spokesperson for Lymphatic Education and Research Network (LE\&RN) a nonprofit organization dedicated to fighting lymphatic diseases and LE. As part of our emphasis on surgical innovation, this year's meeting reprised the John Wayne Cancer Institute's Critical Issues in Breast Cancer Forum, which featured the latest trials of

(C) Society of Surgical Oncology 2019

First Received: 21 June 2019;

Published Online: 24 July 2019

H. M. Kuerer, MD, PhD

e-mail: hkuerer@mdanderson.org cryoablation, robotic nipple-sparing mastectomy, and intraoperative radiotherapy. Furthermore, the first annual Hoag Hospital Foundation Surgical Innovation Forum featured a special lecture presentation by Dr. Melvin J. Silverstein and introduction of the society's exciting new Oncoplastic Surgery Credentialing Program by Dr. Juliann Reiland. In keeping with the theme of innovation, persuasive feature presentations and discussions on other cutting edge topics, including new society consensus guidelines for offering breast cancer patients genetic testing, percutaneous biopsy trials to avoid surgery after neoadjuvant systemic therapy among patients achieving a complete pathologic response to therapy, updates on the leading RTOG and Alliance cooperative group trials, breast cancer immunotherapy, surgeon-performed nerve blocks and pain management, and the ever-popular great debates regarding margins, radiotherapy after neoadjuvant systemic therapy. ${ }^{1}$ Finally, critical topics on ethical issues, genetic testing, use of narcotics for pain control, and new perspectives on management of benign breast diseases were well received.

\section{ORAL AND SCIENTIFIC SESSIONS}

Much gratitude is given to the 27 members of the Publication Committee for careful review of nearly 300 research abstracts for selection of the top studies for oral scientific and "quick-shot" presentations and poster-presentations, and final review and selection of the highest impact publications for this issue of Annals of Surgical Oncology. The scientific quality of work submitted is continuing to increase such that an additional Oral Presentation session was necessary to facilitate important transfer of information to our members regarding critical issues and findings from much more than can be mentioned here in this brief introduction. Just some of the highlighted 
research studies published in this issue of Annals of Surgical Oncology include essential work regarding nipplesparing mastectomy and patient-reported outcomes based on type of breast cancer surgery and reconstruction received. $^{2-8}$ The annual Scientific Presentation Award for the best presentation by a fellow/resident/or trainee went to Dr. Olga Kantor for her work on physician knowledge regarding contralateral prophylactic mastectomy, and both the Scientific Impact Award for the best presentation as voted by the attendees and the George Peter's Award for the best presentation by a fellow went to Dr. Susie Sun for her work on selecting patients for non-operative management clinical trials after neoadjuvant systemic therapy. ${ }^{9,10}$

Finally, ensuring the best quality comprehensive breast care today and the future requires healthy surgeons and teams taking care of our patients. Because breast surgery practice is a high-patient-volume specialty and often associated with managing the understandable high-level of emotional distress of our breast cancer patients, it was not entirely surprising to find that approximately $41 \%$ of our surveyed members expressed burnout (i.e., emotional exhaustion and interpersonal disengagement. ${ }^{11}$ Our work in this arena has just begun and begins by addressing the key reciprocal elements of physician-wellbeing and professional fulfillment: a culture of wellness, personal resilience, and efficiency of practice. ${ }^{12}$ These elements provide our members and organizational practice structures a clear roadmap for success that will continue to be a focus for the Society the next several years.

DISCLOSURE The authors declare no conflict of interest.

\section{REFERENCES}

1. Manahan ER, Kuerer HM, Sebastian M, et al. Consensus Guidelines on Genetic Testing for Hereditary Breast Cancer from the American Society of Breast Surgeons. Ann Surg Oncol. 2019. https://doi.org/10.1245/s10434-019-07549-8.

2. Young WA, Degnim AC, Hoskin TL, et al. Outcomes of $>1,300$ nipple-sparing mastectomies with immediate reconstruction: the impact of expanding indications on complications. Ann Surg Oncol. 2019. https://doi.org/10.1245/s10434-019-07560-z.

3. Kopkash K, Novak K, Kuchta K, et al. The "Nipple Whipple"?! A pilot study to assess the ergonomic effects of nipple sparing mastectomy. Ann Surg Oncol. 2019. https://doi.org/10.1245/s10 434-019-07550-1.

4. Wong SM, Chun YS, Sagara Y, Golshan M, Erdmann-Sager J. National patterns of breast reconstruction and nipple-sparing mastectomy for breast cancer, 2005-2015. Ann Surg Oncol. 2019. https://doi.org/10.1245/s10434-019-07554-x.

5. Flanagan MR, Zabor EC, Romanoff A, et al. A comparison of patient-reported outcomes after breast-conserving surgery and mastectomy with implant breast reconstruction. Ann Surg Oncol. 2019. https://doi.org/10.1245/s10434-019-07548-9.

6. Fan B, Valente SA, Shilad S, et al. Reducing narcotic prescriptions in breast surgery: a prospective analysis. Ann Surg Oncol. 2019. https://doi.org/10.1245/s10434-019-07542-1.

7. Vuong B, Graff-Baker AN, Yanagisawa M, et al. Implementation of a post-mastectomy home recovery program in a large, integrated health care delivery system. Ann Surg Oncol. 2019. https://doi.org/10.1245/s10434-019-07551-0.

8. Keehn AR, Olson DW, Dort JC, et al. Same-day surgery for mastectomy patients in Alberta: A perioperative care pathway and quality improvement initiative. Ann Surg Oncol. 2019. http s://doi.org/10.1245/s10434-019-07568-5.

9. Kantor O, Chang C, Bleicher RJ, et al. Physician knowledge of breast cancer recurrence and contralateral breast cancer risk is associated with increased recommendations for contralateral prophylactic mastectomy: a survey of physicians at NAPBC accredited centers. Ann Surg Oncol. 2019. https://doi.org/10.124 5/s10434-019-07559-6.

10. Sun S, van la Parra RFD, Rauch GM, et al. Patient selection for clinical trials eliminating surgery in HER2-positive breast cancer treated with neoadjuvant systemic therapy. Ann Surg Oncol. 2019. https://doi.org/10.1245/s10434-019-07533-2.

11. Zhang JQ, Riba L, Magrini L, et al. Assessing burnout and professional fulfillment in breast surgery: Results from a national survey of the American Society of Breast Surgeons. Ann Surg Oncol. 2019. https://doi.org/10.1245/s10434-019-07532-3.

12. Bohman B, Dyrbye L, Sinsky CA, et al. Physician well-being: the reciprocity of practice efficiency, culture of wellness, and personal resilience. NEJM Catalyst. Aug 7 2017. https://catalyst.ne jm.org/physician-well-being-efficiency-wellness-resilience/.

Publisher's Note Springer Nature remains neutral with regard to jurisdictional claims in published maps and institutional affiliations. 